\title{
Treatment Considerations of Lung Involvement in Rheumatologic Disease
}

\author{
Alejandro Robles-Perez Maria Molina-Molina \\ ILD Unit, Respiratory Department, University Hospital of Bellvitge, Barcelona, Spain
}

\author{
Key Words \\ Treatment $\cdot$ Interstitial lung disease $\cdot$ Rheumatologic \\ disease $\cdot$ Connective tissue disease
}

\begin{abstract}
Lung involvement in rheumatologic diseases has a broad spectrum of clinical and radiological presentations, from acute and inflammatory predominance to chronic and fibrotic lung disorders. Therapy has to be guided by the type of rheumatologic disease, the kind of lung involvement and the risk associated with the clinical state of the patient and prognostic factors. Although several therapeutic approaches have been used, the best treatment is still not certain; clinical trials are mandatory, which would require a better knowledge of the pulmonary pathogenesis of immunologic diseases. The following review is focused on the therapeutic options for those rheumatologic disorders that could present as a predominant interstitial lung disease which may confer a bad or life-threatening prognosis.
\end{abstract}

(c) 2015 S. Karger AG, Basel

\section{Introduction}

Therapeutic interventions in rheumatologic diseases generally target at least one of the biological processes characteristic of the entity, which usually comprise autoimmunity, inflammatory cell infiltration, tissue fibrosis and, in some cases, vasculopathy. The level of disease activity and the degree of specific organ involvement usu- ally determine treatment decisions. However, there is no clear evidence of overall disease modification in most cases. The main pathogenic targets to treat when chronic and fibrotic lung disease is predominant are epithelial alveolar injury and lung remodeling. If parenchymal lung involvement is acute or subacute, the main process to focus on is inflammatory infiltration as well as alveolar epithelial cell damage. However, some chronic lung disorders can also present acute exacerbations that may require an anti-inflammatory approach based on corticosteroids. Furthermore, most connective tissue diseases (CTD) present some immunologic features and other organ involvement that also should be taken into account. Finally, lung transplantation is an option for patients at the end stage of pulmonary disorder that do not present contraindications.

Thus, the therapeutic approach to the lung is complex and limited by the lack of effective treatments for fibrotic immunologic diseases as well as the low or very low level of evidence for some particularly uncommon rheumatologic diseases. The present review is focused on the treatment approach to nonvascular parenchymal lung diseases associated with rheumatologic entities.

Previous articles in this series: 1. Bouros D, Papiris S, Cottin V: Lung involvement in rheumatic disease: introduction. Respiration 2015;90:1. 2. Papiris SA, Manali ED, Kolilekas L, Kagouridis K, Maniati M, Borie R, Pradere $P$, Crestani $B$, Bouros D: Investigation of lung involvement in connective tissue disorders. Respiration 2015;90:2-24. 3. Fischer A, Lee JS, Cottin V: Interstitial lung disease evaluation: detecting connective tissue disease. Respiration 2015;90:177-185.

\section{KARGER 125}

C 2015 S. Karger AG, Basel

0025-7931/15/0904-0265\$39.50/0
Maria Molina-Molina, $\mathrm{MD}, \mathrm{PhD}$

ILD Unit, Respiratory Department

University Hospital of Bellvitge, c/Feixa Llarga, s/n

ES-08907 Hospitalet de Llobregat, Barcelona (Spain)

E-Mail mariamolina@bellvitgehospital.cat 


\section{Systemic Sclerosis}

Systemic sclerosis (SS) is a relatively rare autoimmune disorder associated with a high prevalence of lung disease, especially in the form of interstitial lung disease (ILD) [1]. Although the most predominant radiologicalhistological pattern is nonspecific interstitial pneumonia (NSIP), usual interstitial pneumonia (UIP) can be present and is usually associated with the poorest prognosis [1]. The level of evidence to establish a treatment for SS-associated ILD (SS-ILD) is low, but SS-ILD has been more extensively studied than other CTD. The therapeutic approach to SS-ILD includes (1) treatment of the symptoms, such as cough relief with codeine or other minor opioids; (2) treatment of associated factors that may contribute to ILD progression, such as gastroesophageal reflux disease by following a diet (low-fat meals, small quantities, no alcohol, soda or other sparkling drinks), elevating the head of the bed and taking proton pump inhibitors, and (3) treatment with drugs that are said to inhibit ILD progression, which will be covered in depth below. Most of the evidence that suggests a benefit from these agents is based on small population studies; thus, well-designed clinical trials are required to better define the role of these agents in SS-ILD [2].

\section{Cyclophosphamide}

Cyclophosphamide is the most studied drug for the treatment of SS-ILD. Several studies have been conducted, including two prospective multicenter placebo-controlled randomized clinical trials. The North American Scleroderma Lung study assessed the efficacy of 12 months of oral cyclophosphamide, $<2 \mathrm{mg} / \mathrm{kg} /$ day, versus placebo in 158 patients with SS-ILD [2]. The treatment arm presented a statistically significant prevention of forced vital capacity (FVC) decline compared with the placebo arm ( $\mathrm{p}<0.03)$ [3]. Improvement of dyspnea, health-related quality of life and skin thickness was also observed. In the study by Hoyles et al. [4], 45 patients were recruited to receive for 6 months $20 \mathrm{mg}$ oral prednisolone on alternate days and monthly intravenous (i.v.) cyclophosphamide $\left(600 \mathrm{mg} / \mathrm{m}^{2}\right.$, mean dose 1,050 $\mathrm{mg})$, followed by daily oral azathioprine $(2.5 \mathrm{mg} / \mathrm{kg} /$ day $)$ as maintenance therapy, versus matched placebo treatment. At 12 months, a trend toward a statistically significant difference in FVC decline was observed in the treatment arm compared with the placebo arm $(\mathrm{p}=0.08)$. Particularly, the outcomes of both studies suggest that the treatment effect lies in the reduction of fibrotic SSILD progression.
Nadashkevich et al. [5] performed an unblinded randomized trial comparing cyclophosphamide and azathioprine. Patients treated with cyclophosphamide presented an increase of $3.3 \%$ in FVC, while those treated with azathioprine showed a decrease in FVC $(-11.1 \%$; $\mathrm{p}<$ 0.001). A meta-analysis [6] including the mentioned clinical trials and six prospective observational studies concluded that cyclophosphamide inhibited a decline in FVC and diffusing capacity of the lung for carbon monoxide (DLCO), but the authors suggested that this was not clinically relevant. Oral treatment, which is as effective as intravenous administration, seems to cause more adverse events, such as leukopenia, neutropenia and bladder cancer $[3,7]$. According to their data, the EULAR Scleroderma Trials and Research (EUSTAR) group concluded that cyclophosphamide should be considered for SS-ILD treatment, tailoring doses and durations individually [8].

\section{Azathioprine}

In the study by Nadashkevich et al. [5] mentioned above, cyclophosphamide showed better results than azathioprine as induction therapy for SS-ILD. A recent retrospective study [9] comparing 15 patients treated with azathioprine and 21 patients that received oral cyclophosphamide during 6 months showed a significant benefit in FVC and DLCO in the azathioprine subgroup. This subgroup had a longer duration of disease, had more groundglass patterns on high-resolution computed tomography (HRCT) and was older at the time of diagnosis. This would suggest that azathioprine could be useful in a selected group of patients. The effect of azathioprine as maintenance treatment for SS-ILD after induction with cyclophosphamide has been studied. Some authors [1012] suggested that treatment with azathioprine in cyclophosphamide responders is associated with stabilization of lung function, quality of life and skin alterations.

\section{Mycophenolate Mofetil}

Mycophenolate is a well-tolerated drug traditionally used as maintenance therapy for SS-ILD patients. A meta-analysis [13] including 69 patients found that mycophenolate could stabilize the lung impairment without significant side effects. A retrospective study [14] including 44 patients with SS-ILD treated with mycophenolate demonstrated stabilization of FVC and DLCO and a reduction in corticosteroid requirement with acceptable tolerance. Two studies on the use of mycophenolate in cyclophosphamide nonresponders were performed [10, 15]. Only one of them showed stabilization in pulmonary 
Table 1. Suggested dosages for every medication, based on the published studies

\begin{tabular}{ll}
\hline Medication [Ref.] & Dosage \\
\hline Prednisone [2, 24, 90] & $\begin{array}{l}0.5-1 \mathrm{mg} / \mathrm{kg} / \text { day up to } 60 \mathrm{mg} / \text { day orally, } 3-8 \text { weeks and } \\
\text { progressive tapering }\end{array}$ \\
Methylprednisolone [2, 24, 90] & $1,000 \mathrm{mg} /$ day i.v. for 3 days ${ }^{\mathrm{a}}$ \\
Cyclophosphamide $[5-11]$ & $1-2 \mathrm{mg} / \mathrm{kg} /$ day orally; $500-1,000 \mathrm{mg}$ i.v. every 4 weeks \\
Azathioprine $[4,9,12]$ & $1-2 \mathrm{mg} / \mathrm{kg} /$ day orally \\
Mycophenolate mofetil $[13,14,91]$ & $720-2,000 \mathrm{mg} /$ day orally \\
Rituximab [21, 26, 39, 40] & $1,000 \mathrm{mg}$ i.v. every $2-4$ weeks \\
Abatacept [25, 92, 93] & $2-10 \mathrm{mg} / \mathrm{kg}$ i.v. every 2 weeks (3 infusions), followed by infusions \\
& every $4 \mathrm{weeks}$ \\
Cyclosporin A [86] & $2-3 \mathrm{mg} / \mathrm{kg} /$ day orally \\
Tacrolimus [87] & $1-3 \mathrm{mg} /$ day orally \\
\hline
\end{tabular}

Some drugs have been tested at different doses and with different types of administration. Furthermore, doses can differ depending on the clinicoradiological presentation, severity and prognostic factors for every patient.

${ }^{a}$ First days of acute exacerbation.

function tests (PFT) [15]. In a consensus of experts [16], mycophenolate was chosen as the preferred maintenance treatment by $73 \%$ of participants. A retrospective study that included 10 patients, comparing the effect of mycophenolate with cyclophosphamide as induction treatment for SS-ILD, found no differences in PFT [17]. Currently, the effect of mycophenolate as first-line treatment compared with cyclophosphamide is being evaluated in the Scleroderma Lung Study II (NCT00883129).

\section{Rituximab}

The use of this chimeric anti-CD20 monoclonal antibody in SS-ILD has been tested with promising results but with a limited number of patients and a low level of evidence. In two studies $[18,19]$ of the same cohort including 14 patients ( 6 treated with rituximab and 8 with other immunosuppressive treatments), a significant difference in FVC and DLCO change in favor of the rituximab arm was observed at 1 and 2 years. Bosello et al. [20] demonstrated that 9 cyclophosphamide nonresponders presented FVC stabilization after one course of rituximab. Jordan et al. [21] assessed the effects of rituximab in the EUSTAR cohort. Nine patients with SS-ILD receiving rituximab presented stabilization in FVC compared with other treatments $(p=0.02)$. In a study of 20 patients with diffuse SS, rituximab improved FVC values in the whole cohort, with FVC stabilization in the SS-ILD subgroup [22]. A clinical trial is now being conducted to compare cyclophosphamide and rituximab in CTD-ILD, including SS-ILD (NCT01862926). The dose administration has been summarized in table 1 .

Treatment Considerations of Lung Involvement in Rheumatologic Disease

\section{Corticosteroids}

Low-dose corticosteroids are one of the first-line treatments for SS-ILD [2]. This common use is controversial due to the risk of acute renal crisis associated with their use and the lack of clinical trials that demonstrate a benefit in SS-ILD [2]. Therefore, oral corticosteroids are usually avoided in patients with fibrotic SS-ILD [2, 23]. However, a corticosteroid response might be observed in nonfibrotic SS-ILD such as cellular NSIP and organizing pneumonia (OP) [23]. Furthermore, acute exacerbations can be present in SS-ILD [23]. Following the recommendations for the treatment of acute exacerbations in idiopathic pulmonary fibrosis (IPF), corticosteroids are often prescribed, despite very limited evidence, after sampling for bacterial cultures and initiation of broad-spectrum antibiotics [24]. The dose and duration will depend on the severity and clinicoradiological follow-up (table 1).

\section{Other Treatments}

Tocilizumab, an anti-interleukin-6 receptor antibody, and abatacept (table 1), an inhibitor of T-cell lymphocyte activation, have recently been tested in different types of scleroderma, mainly in some refractory cases. However, the safety of the two drugs in SS has not yet been demonstrated [25]. Furthermore, no trend for any change of fibrotic lesions has been seen until now [25]. Intravenous immunoglobulin may also be an effective therapy for some forms of refractory SS, but no benefit in SS-ILD has been tested [26]. Hematopoietic stem cell transplantation has been evaluated in two controlled clinical trials in comparison with cyclophosphamide [27, 28]. Both stud- 
Fig. 1. Optional treatment algorithm for SS; fibrotic SS-ILD and nonfibrotic SS-ILD. Note that rational and alternative options could also be possible, depending on the practice at each ILD center. When SS-ILD is progressive, mainly in fibrotic SS-ILD, lung transplantation should always be considered for patients without exclusion criteria and treatment nonresponse.

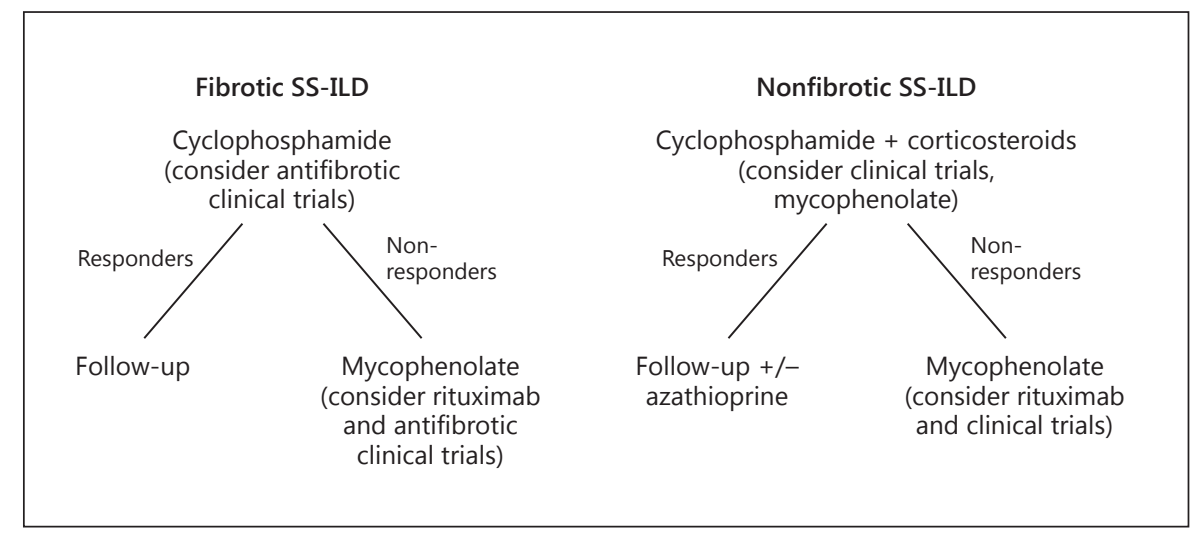

ies showed a significant benefit in FVC compared with cyclophosphamide, and one of them showed a decrease in the volumetric measurement of ILD on HRCT. Stem cell transplantation is a complex and expensive treatment that requires better study, but it might be a promising therapeutic approach for selected patients. Finally, the safety and tolerability of pirfenidone, the first antifibrotic drug that demonstrated a benefit in IPF, are being studied in a clinical trial for SS-ILD patients that present a UIP pattern (LOTUSS, NTC01933334).

\section{Treatment Considerations in SS}

The decision of treating SS-ILD should be taken in those patients at risk of progressive lung disease, depending on symptoms and severity. Several factors have been associated with progressive ILD, such as male sex, AfricanAmerican ethnicity [29, 30], FVC and DLCO decrease [31] and evidence of irreversible fibrotic signs on HRCT such as honeycombing [32]. The treatment for SS-ILD should be chosen individually for every patient and decided on by a multidisciplinary expert team. The radiological pattern of UIP and some fibrotic NSIP on HRCT not only is a bad prognostic factor, but also would require an antifibrotic approach that is not currently well established for this and other rheumatologic diseases [2]. New antifibrotic lung drugs, initially tested in IPF, could be considered as possible future treatment in cases of fibrotic SS-ILD. According to the limited evidence available, until now we suggest the following algorithm as a treatment option (fig. 1), although it may differ from the practice at many ILD centers and from other previously published algorithms. Finally, the possible existence of pulmonary arterial hypertension (PAH) should always be considered in SS, since the presence of this disorder, together with SS-ILD or alone, is a frequent cause of dyspnea and hypoxemia [2].

\section{Rheumatoid Arthritis}

Rheumatoid arthritis (RA) is the most common CTD; pulmonary manifestations are common and cause up to $20 \%$ of overall mortality [2]. Despite this fact, the evidence for treatments for this condition is of low quality. Furthermore, the complex differential diagnosis - with drug-induced ILD related to treatment for RA (methotrexate, etanercept, gold salts and sulfasalazine) and the risk of respiratory infection, especially in RA-related bronchiectasis patients - needs to be taken into account when evaluating these patients [2]. Pharmacological treatment of RA will depend on the type and grade of the associated ILD.

\section{Corticosteroids}

Treatment with corticosteroids $(0.25-1 \mathrm{mg} / \mathrm{kg} /$ day $)$ is still the mainstay of therapy, chiefly based on evidence from some idiopathic interstitial pneumonia cases and the fact that this treatment is also useful for other affected organs or tissues. In patients with RA-ILD, OP and NSIP patterns respond better to corticosteroids [33]. However, the effect on patients with a UIP pattern remains unclear. The retrospective study by Song et al. [34] included 84 RA patients that presented a UIP pattern; $50 \%$ of the patients were treated with corticosteroids in association with different immunosuppressors and presented an improvement or stabilization of lung function, but no significant difference was found from the untreated group.

\section{Immunosuppressive Therapy}

Reports on potential benefits from cyclophosphamide, azathioprine and cyclosporine have been published [34-37] (table 1). Nevertheless, there is no strong 
evidence for their recommendation for RA-ILD. On the other hand, a longitudinal retrospective study showed that the use of mycophenolate mofetil combined with a low dose of prednisone was associated with improvement in PFT in patients with an RA-associated non-UIP pattern and with stabilization in those with a UIP pattern [14]. However, no placebo arm was included in the study. Furthermore, clinical improvement with lung functional and radiological stabilization has also been described [38].

Rituximab is considered a rescue therapy in RA-ILD, although based on a very low level of evidence [39]. Several case reports focusing on the use of rituximab in RAILD patients have shown stabilization or improvement of the lung disease $[39,40]$. However, these patients received the treatment as a second- or third-line treatment. A recent clinical trial focusing on patients with NSIP or UIP patterns has shown stabilization or improvement in PFT, imaging studies, joint symptoms and quality of life in 6 out of 7 patients [41]. The safety and tolerability of rituximab has been tested, and only induced inflammatory disorders that improved with corticosteroids have been reported [42-45]. Based on these results, some groups initiate rituximab when corticosteroids and other immunosuppressors fail to inhibit the progression of RA-ILD. Nevertheless, clinical trials are required to test the benefit from this drug in RA-ILD.

\section{Drug-Induced Lung Disease Related to RA Treatment}

Drug toxicity is an important issue that should always be taken into account when evaluating patients with lung disease in the context of RA. Several disease-modifying antirheumatic drugs (DMARD) have been shown to be involved in the development of lung disease [2]. In most cases, it is difficult to differentiate RA-ILD from drug toxicity, as the diagnosis of ILD often comes after years of treatment, when the lung damage is already established. Imaging findings are usually nonspecific, with diffuse pulmonary opacities or patchy consolidation on HRCT, and the study of biological samples is more useful for ruling out infection than for confirming the diagnosis [46]. Drug-induced ILD should be suspected when clinical, functional or radiological abnormalities appear in the first 6 months of treatment. In this scenario, differentiating drug-induced ILD from infection, exacerbation of underlying ILD or RA-ILD progression becomes a challenge for the physician [47]. Therefore, a functional and radiological evaluation of the lung should be considered prior to starting a new RA treatment [48, 49].

Treatment Considerations of Lung Involvement in Rheumatologic Disease

\section{Nonbiological DMARD}

Many cases of methotrexate-related lung disease have been described [50]. Methotrexate can exacerbate a preexisting lung disease or develop new lung impairment. The American College of Rheumatology recommends avoiding methotrexate in patients with RA and symptomatic ILD [49]. Despite the common association of methotrexate with lung disease, a recent meta-analysis found no differences in the presence of pneumonitis between patients that received methotrexate and those that received other agents [50]. Risk factors for the development of methotrexate-related lung disease have been described: older age, diabetes, hypoalbuminemia, extra-articular manifestations and previous use of other DMARD [51].

Leflunomide has been less associated with exacerbation of preexisting ILD or development of new lung disease. The principal risk factors are preexisting lung disorder and previous use of methotrexate. If these conditions are not present, the incidence of leflunomide-related ILD is rare [52-54].

\section{Biological DMARD}

TNF- $\alpha$ inhibitors have been shown to be involved in exacerbation and new onset of ILD. Panopoulos and Sfikakis [55] described 144 patients with development or exacerbation of ILD after treatment with these drugs. Another series showed 108 RA patients who developed ILD after treatment with infliximab or etanercept. Longstanding ILD was associated with risk of death [56]. Despite these case reports, other cohort studies failed to show higher rates of ILD among patients treated with TNF inhibitors in comparison with other treatments [57, 58].

\section{Treatment Considerations in RA}

Pulmonary toxicity from methotrexate, leflunomide or TNF inhibitors has been estimated at around 1\% each $[2,46]$. Thus, avoiding these treatments without any evidence of lung disease is not recommended. Performing a lung evaluation prior to treatment of RA could be of interest in that patients with RA-ILD might be detected [59]. In the setting of subclinical RA-ILD, these drugs have shown a heightened risk of causing ILD progression. Thus, lung function monitoring during treatment would be useful in order to detect lung disease development or progression early.

Infections of the lower respiratory tract in RA-ILD require special consideration when increases in dyspnea or hypoxemia and/or new radiological infiltrates appear [2]. 
Fig. 2. Suggested treatment options for RAassociated lung disease. Lung transplantation is considered for nonresponding progressive RA-ILD patients, which should occur mainly in cases of UIP and fibrotic NSIP RA-ILD. MTX = Methotrexate.

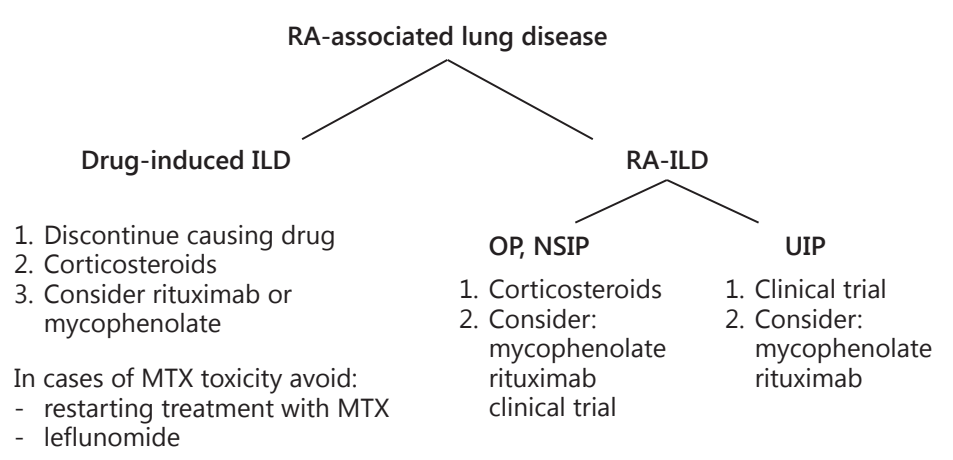

A broad spectrum of antibiotics, including co-trimoxazole for Pneumocystis jiroveci, is indicated, and even mandatory in order to discharge mycobacteria and other opportunistic infections, principally when patients have received DMARD treatment $[2,23]$.

In cases of progressive RA-ILD, mycophenolate and rituximab have shown promising efficacy and safety, though further placebo-controlled trials need to be performed in this group of patients. If drug-induced ILD is detected, drug discontinuation and use of high-dose corticosteroids is recommended. In cases of methotrexate toxicity, restarting the drug or treatment with leflunomide after resolution of the ILD should be avoided [46, 60]. A suggested treatment option according to the data available is shown in figure 2 .

\section{Sjögren Syndrome}

Lung disease in Sjögren syndrome appears in 10-20\% of the cases. It can present in different ways, especially as distal airway disease and ILD, including NSIP, OP, lymphocytic interstitial pneumonitis, primary pulmonary lymphoma and, less frequently, UIP, as well as diffuse interstitial amyloidosis [61]. Evidence for treatments of this disease comes from case reports and small series, and the pharmacological therapy has usually been extrapolated from the treatment of idiopathic interstitial pneumonias.

\section{Corticosteroids}

Corticosteroids are commonly used as first-line therapy. Corticosteroids alone or in combination with immunosuppressive therapies have demonstrated benefits in treating Sjögren syndrome-associated bronchiolitis and
ILD, mainly for OP [62-64]. In the study by Parambil et al. [62], 72\% of those patients treated with corticosteroids alone or in combination with immunosuppressive therapy presented stabilization or improvement of lung function. In another series, $63 \%$ of the patients treated with azathioprine alone or in combination with prednisone presented functional improvement [64].

\section{Rituximab}

Rituximab seems to be a safe and useful drug for the treatment of Sjögren syndrome, especially in cases with systemic involvement. In the setting of Sjögren syndrome-related ILD, improvement in lung disease has been observed in small series of cases [65-67].

\section{Treatment Considerations for Sjögren Syndrome}

The data regarding treatment of Sjögren syndromerelated lung disease are of low quality. In this particular disease, since it is associated more frequently than others with lymphocytic interstitial pneumonitis and potential subsequent lymphoma, a differential diagnosis of lung infiltrates on HRCT is of significant importance. The firstline therapy consists of corticosteroids alone or in combination with azathioprine or other immunosuppressors. In refractory cases, treatment with rituximab may be considered. Sjögren syndrome may coexist with other autoimmune rheumatologic diseases, which could influence therapeutic decisions.

\section{Systemic Lupus Erythematosus}

Lung disease in systemic lupus erythematosus (SLE) involves different parts of the respiratory system. Pleural disease, pneumonitis and diffuse alveolar hemorrhage are 
the most common manifestations, but other ILD, the shrinking lung syndrome and PAH may also be present. In fact, pleuritis is a diagnostic criterion for SLE [68], and treatment is based on nonsteroidal anti-inflammatory drugs, as well as on corticosteroids for refractory cases [69]. PAH-SLE should be suspected mainly in antiphospholipid syndrome [68]. Lupus pneumonitis, the other typical pulmonary manifestation, usually requires high doses of corticosteroids [69]. Lower respiratory tract infection should be considered before the initiation of corticosteroids.

\section{Interstitial Lung Disease}

The evidence for treatments of SLE-ILD is of low quality, and mainly based on expert opinion. A recent consensus on the treatment of SLE proposed induction therapy with corticosteroids alone or in association with cyclophosphamide or mycophenolate mofetil, and maintenance therapy with azathioprine or mycophenolate [70]. As second-line therapy, the use of rituximab was suggested [70].

Diffuse alveolar hemorrhage is a life-threatening condition with a mortality rate of $50 \%$ [71]. Standard treatment is based on pulse methylprednisolone ( $1 \mathrm{~g}$ daily for $72 \mathrm{~h}$ ) followed by prednisone (1-2 mg/kg/day) [72]. Successful treatment with mycophenolate mofetil, rituximab or plasmapheresis in refractory cases has been described [73-75].

\section{Shrinking Lung Syndrome}

Management of shrinking lung syndrome is based on case series. No clinical trials have been conducted, as it is an uncommon manifestation of this rare disease. Traditional management includes corticosteroid therapy alone or in association with immunosuppressive therapy (cyclophosphamide, azathioprine or mycophenolate mofetil). $\beta_{2}$-Agonists, theophylline and even a surgical approach have also been used to increase diaphragmatic strength. In refractory cases, treatment with rituximab has shown promising results [76-79].

\section{Polymyositis and Dermatomyositis}

Inflammatory myopathies express lung disease mainly in the form of ILD. Two clinical presentations have been described: one with a chronic onset and another one with rapidly progressive lung impairment and a mortality rate of up to $50 \%[80]$.

Treatment Considerations of Lung Involvement in Rheumatologic Disease

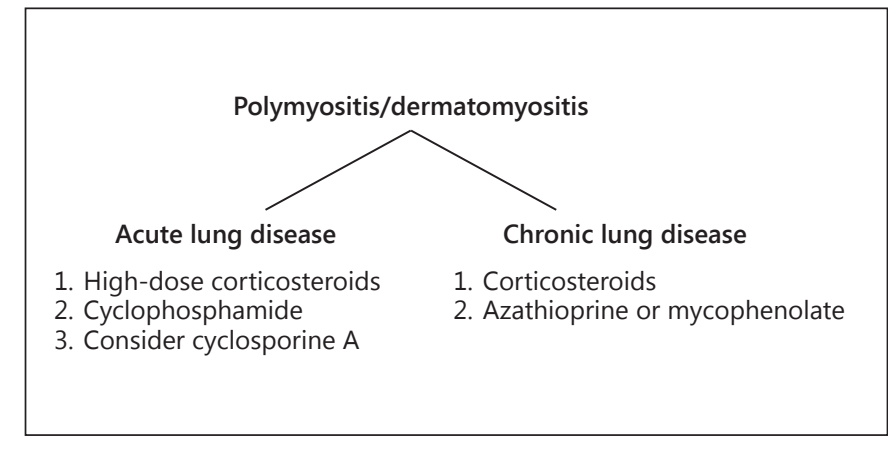

Fig. 3. Proposed treatment algorithm for polymyositis/dermatomyositis.

\section{Corticosteroids}

Corticosteroids, either orally or intravenously, are still the first-line therapy. Daily intravenous infusions of $1 \mathrm{~g}$ methylprednisolone for 1-3 days followed by $0.5-1 \mathrm{mg} /$ $\mathrm{kg} /$ day oral prednisone with progressive tapering are a suggested regimen of treatment $[80,81]$. The clinical response in chronic ILD is better than in cases of rapidly progressive disease, when additional immunotherapy is often required $[82,83]$.

\section{Cyclophosphamide}

Cyclophosphamide has shown better results in the treatment of rapidly progressive ILD, with improvement or stabilization of lung deterioration [84]. However, only $55 \%$ of cases have a benefit in the chronic form of the disease [82].

\section{Azathioprine}

The use of this drug has shown improvement or stabilization of the chronic presentation of ILD in $58 \%$ of cases when receiving concomitant treatment with corticosteroids or after induction with cyclophosphamide [85].

\section{Calcineurin Inhibitors}

Cyclosporin A has shown some clinical response in both chronic and rapidly progressive forms of ILD [83, 86]. The addition of tacrolimus to standard therapy with corticosteroids, cyclophosphamide and/or cyclosporine has led to an improvement in prognosis in either acute or chronic onset of ILD in a retrospective study [87].

\section{Mycophenolate Mofetil}

Treatment with mycophenolate as first- or second-line therapy has shown stabilization or improvement of ILD in the chronic form of the disease in up to $80 \%$ of patients 
in some case series $[82,88]$. Despite the limited evidence due to its safety profile, mycophenolate is used as a steroid-sparing therapy.

\section{Rituximab}

In a series of 11 patients treated with anti-aminoacyltRNA synthetase antibodies, 63\% presented improvement or stabilization of ILD after treatment with rituximab as second-line therapy [89].

\section{Intravenous Immunoglobulins}

Although intravenous immunoglobulins seem to be useful in refractory polymyositis, the effect on lung involvement remains controversial $[84,88]$.

\section{Treatment Considerations for Polymyositis/ \\ Dermatomyositis-ILD}

Treatment for this condition should be started in symptomatic or progressive forms of the disease. Firstline treatment consists of corticosteroids. The use of concomitant treatments is based on low-quality evidence and should be reserved for the most severe cases and as a corticosteroid-sparing treatment. In cases of acute ILD, in- tensive therapy and the option of lung transplantation should be considered as soon as in other CTD-ILD, since it is associated with a high short-term mortality. Finally, the possibility of cancer-related polymyositis/dermatomyositis merits special consideration in some cases. A suggested therapeutic approach is shown in figure 3.

\section{Mixed CTD}

This overlapping systemic disease presents with respiratory involvement in up to $80 \%$ of patients. The main respiratory manifestations are ILD, including pulmonary fibrosis (fibrotic NSIP and UIP patterns), pleural effusion and PAH [2]. As in other rheumatologic diseases, no controlled data exist on which to base the therapy for interstitial lung involvement in mixed CTD.

Most ILD are treated with corticosteroids and/or immunosuppressive drugs, which eventually inhibit autoimmune-driven mononuclear cell infiltration in the lung parenchyma. The regimens used for ILD in SS appear appropriate and usually consist of low-dose corticosteroids in combination with cyclophosphamide or azathioprine [2].

\section{References}

$>1$ Bouros D, Wells AU, Nicholson AG, et al: Histopathologic subsets of fibrosing alveolitis in patients with systemic sclerosis and their relationship to outcome. Am J Respir Crit Care Med 2002;165:1581-1586.

$>2$ Antoniou KM, Margaritopoulos G, Economidou F, et al: Pivotal clinical dilemmas in collagen vascular diseases associated with interstitial lung involvement. Eur Respir J 2008;33: 882-896.

3 Tashkin DP, Elashoff R, Clements PJ, et al; Scleroderma Lung Study Research Group: Cyclophosphamide versus placebo in scleroderma lung disease. N Engl J Med 2006;354: 2655-2666.

4 Hoyles RK, Ellis RW, Wellsbury J, et al: A multicenter, prospective, randomized, double-blind, placebo-controlled trial of corticosteroids and intravenous cyclophosphamide followed by oral azathioprine for the treatment of pulmonary fibrosis in scleroderma. Arthritis Rheum 2006;54:3962-3970.

5 Nadashkevich O, Davis P, Fritzler M, et al: A randomized unblinded trial of cyclophosphamide versus azathioprine in the treatment of systemic sclerosis. Clin Rheumatol 2006;25: 205-212.

6 Nannini C, West CP, Erwin PJ, et al: Effects of cyclophosphamide on pulmonary function in patients with scleroderma and interstitial lung disease: a systematic review and metaanalysis of randomized controlled trials and observational prospective cohort studies. Arthritis Res Ther 2008;10:R124.

7 Guillevin L, Cordier JF, Lhote F, et al: A prospective, multicenter, randomized trial comparing steroids and pulse cyclophosphamide versus steroids and oral cyclophosphamide in the treatment of generalized Wegener's granulomatosis. Arthritis Rheum 1997;40:21872198.

$\checkmark 8$ Kowal-Bielecka O, Landewé R, Avouac J, et al; EUSTAR Co-Authors: EULAR recommendations for the treatment of systemic sclerosis: a report from the EULAR Scleroderma Trials and Research Group (EUSTAR). Ann Rheum Dis 2009;68:620-628.

$\checkmark 9$ Poormoghim H, Rezaei N, Sheidae Z, et al: Systemic sclerosis: comparison of efficacy of oral cyclophosphamide and azathioprine on skin score and pulmonary involvement - a retrospective study. Rheumatol Int 2014;34: 1691-1699.

10 Iudici M, Cuomo G, Vettori S, et al: Lowdose pulse cyclophosphamide in interstitial lung disease associated with systemic sclerosis (SSc-ILD): efficacy of maintenance immunosuppression in responders and non- responders. Semin Arthritis Rheum 2015;44: 437-444.

11 Bérezné A, Ranque B, Valeyre D, et al: Therapeutic strategy combining intravenous cyclophosphamide followed by oral azathioprine to treat worsening interstitial lung disease associated with systemic sclerosis: a retrospective multicenter open-label study. J Rheumatol 2008;35:1064-1072.

12 Paone C, Chiarolanza I, Cuomo G, et al: Twelve-month azathioprine as maintenance therapy in early diffuse systemic sclerosis patients treated for 1 year with low dose of cyclophosphamide pulse therapy. Clin Exp Rheumatol 2007;25:613-616.

13 Tzouvelekis A, Galanopoulos N, Bouros E, et al: Effect and safety of mycophenolate mofetil or sodium in systemic sclerosis-associated interstitial lung disease: a meta-analysis. Pulm Med 2012;2012:143637.

14 Fischer A, Brown KK, Du Bois RM, et al: Mycophenolate mofetil improves lung function in connective tissue disease-associated interstitial lung disease. J Rheumatol 2013;40:640646.

15 Yilmaz N, Can M, Kocakaya D, et al: Twoyear experience with mycophenolate mofetil in patients with scleroderma lung disease: a case series. Int J Rheum Dis 2014;17:923-928. 
16 Walker KM, Pope J; Participating Members of the Scleroderma Clinical Trials Consortium; Canadian Scleroderma Research Group: Treatment of systemic sclerosis complications: what to use when first-line treatment fails - a consensus of systemic sclerosis experts. Semin Arthritis Rheum 2012;42:42-55.

-17 Panopoulos ST, Bournia VK, Trakada G, et al: Mycophenolate versus cyclophosphamide for progressive interstitial lung disease associated with systemic sclerosis: a 2-year case control study. Lung 2013;191:483-489.

18 Daoussis D, Liossis SN, Tsamandas AC, et al: Experience with rituximab in scleroderma: results from a 1-year, proof-of-principle study. Rheumatology 2010;49:271-280.

19 Daoussis D, Liossis SN, Tsamandas AC, et al: Effect of long-term treatment with rituximab on pulmonary function and skin fibrosis in patients with diffuse systemic sclerosis. Clin Exp Rheumatol 2012;30(suppl 71):S17-S22.

20 Bosello S, De Santis M, Lama G, et al: B cell depletion in diffuse progressive systemic sclerosis: safety, skin score modification and IL-6 modulation in an up to thirty-six months follow-up open-label trial. Arthritis Res Ther 2010;12:R54.

21 Jordan S, Distler JHW, Maurer B, et al: Effects and safety of rituximab in systemic sclerosis: an analysis from the European Scleroderma Trial and Research (EUSTAR) group. Ann Rheum Dis 2015;74:1188-1194.

22 Bosello S, De Luca G, Rucco M, et al: Longterm efficacy of B cell depletion therapy on lung and skin involvement in diffuse systemic sclerosis. Semin Arthritis Rheum 2015;44: 428-436.

23 Papiris SA, Kagouridis K, Papadaki G, Kolilekas L, Manali ED: Treating CTDs related fibrotic ILDs by immunosuppressants: 'facts and faults'. Lung 2014;192:221-223.

24 Ryerson CJ, Cottin V, Brown KK, Collard HR: Acute exacerbation of idiopathic pulmonary fibrosis: shifting the paradigm. Eur Respir J 2015; $46: 512-520$.

-25 Elhai M, Meunier M, Matucci-Cerinic M, Maurer B, et al; EUSTAR (EULAR Scleroderma Trials and Research Group): Outcomes of patients with systemic sclerosis-associated polyarthritis and myopathy treated with tocilizumab or abatacept: a EUSTAR observational study. Ann Rheum Dis 2013;72:12171220.

26 McMahan ZH, Wigley FM: Novel investigational agents for the treatment of scleroderma. Expert Opin Investig Drugs 2014;23:183198.

27 Burt RK, Shah SJ, Dill K, et al: Autologous non-myeloablative haemopoietic stem-cell transplantation compared with pulse cyclophosphamide once per month for systemic sclerosis (ASSIST): an open-label randomized phase 2 trial. Lancet 2011;378:498-506.

28 van Laar MJ, Farge D, Sont JK, et al: The ASTIS trial: autologous stem cell transplantation versus iv pulse cyclophosphamide in poor prognosis systemic sclerosis, first results.
EULAR, Annual Congress of the European League against Rheumatism. Ann Rheum Dis 2012;71(suppl 3):51.

29 Barnett AJ: Scleroderma (progressive systemic sclerosis): progress and course based on a personal series of 118 cases. Med J Aust 1978; 2:129-134.

30 Greidinger EL, Flaherty KT, White B, et al: African-American race and antibodies to topoisomerase I are associated with increased severity of scleroderma lung disease. Chest 1998;114:801-807.

31 Plastiras SC, Karadimitrakis SP, Ziakas PD, et al: Scleroderma lung: initial forced vital capacity as predictor of pulmonary function decline. Arthritis Rheum 2006;55:598-602.

32 Khanna D, Tseng CH, Farmani N, et al: Clinical course of lung physiology in patients with scleroderma and interstitial lung disease. Arthritis Rheum 2011;63:3078-3085.

33 Hallowell RW, Horton MR: Interstitial lung disease in patients with rheumatoid arthritis: spontaneous and drug induced. Drugs 2014; 74:443-450.

34 Song J-W, Lee H-K, Lee CK, et al: Clinical course and outcome of rheumatoid arthritisrelated usual interstitial pneumonia. Sarcoidosis Vasc Diffuse Lung Dis 2013;30:103112.

35 Ogawa D, Hashimoto H, Wada J, et al: Successful use of cyclosporine A for the treatment of acute interstitial pneumonitis associated with rheumatoid arthritis. Rheumatology (Oxford) 2000;39:1422-1424.

36 Chang HK, Park W, Ryu DS: Successful treatment of progressive rheumatoid interstitial lung disease with cyclosporine: a case report. J Korean Med Sci 2002;17:270-273.

37 Puttick MP, Klinkhoff AV, Chalmers A, et al: Treatment of progressive rheumatoid interstitial lung disease with cyclosporine. J Rheumatol 1995;22:2163-2165.

38 Saketkoo LA, Espinoza LR: Rheumatoid arthritis interstitial lung disease: mycophenolate mofetil as antifibrotic and disease-modifying antirheumatic drug. Arch Intern Med 2008;168:1718-1719.

39 Braun-Moscovici Y, Butbul-Aviel Y, Guralnik L, et al: Rituximab: rescue therapy in lifethreatening complications or refractory autoimmune diseases: a single center experience. Rheumatol Int 2013;33:1495-1504.

40 Braun MG, Wagener P: Regression of peripheral and pulmonary rheumatoid nodules under therapy with rituximab (in German). Z Rheumatol 2013;72:166-171.

41 Matteson E: Rituximab in rheumatoid arthritis lung disease. NCT00588565. https://www. clinicaltrials.gov/ct2/show/study/NCT00578 565 ? term $=$ NCT00578565\&rank $=1 \&$ sect $=X c$ ba9870156 (updated September 25, 2012).

42 Lioté H, Lioté F, Séroussi B, et al: Rituximabinduced lung disease: a systematic literature review. Eur Respir J 2010;35:681-687.

43 Emery P, Fleischmann R, Filipowicz-Sosnowska $\mathrm{A}$, et al: The efficacy and safety of rituximab in patients with active rheumatoid arthritis despite methotrexate treatment: results of a phase IIB randomized, double-blind, placebo-controlled, dose-ranging trial. Arthritis Rheum 2006;54:1390-1400.

44 Dass S, Atzeni F, Vital E, et al: Safety of rituximab in patients with rheumatoid arthritis and concomitant lung disease. Ann Rheum Dis 2011;70(suppl 3):71.

45 Becerra G, Cambridge M: Safety and efficacy of rituximab in patients with rheumatoid arthritis and lung involvement. Ann Rheum Dis 2013;72(suppl 3):450.

46 Roubille C, Haraoui B: Interstitial lung diseases induced or exacerbated by DMARDs and biologic agents in rheumatoid arthritis: a systematic literature review. Semin Arthritis Rheum 2014;43:613-626.

47 Doyle TJ, Hunninghake GM, Rosas IO: Subclinical interstitial lung disease: why you should care. Am J Respir Crit Care Med 2012; 185:1147-1153.

48 Saravanan V, Kelly CA: Reducing the risk of methotrexate pneumonitis in rheumatoid arthritis. Rheumatology (Oxford) 2009;48: 1065-1068.

49 Saag KG, Teng GG, Patkar NM, et al; American College of Rheumatology: American College of Rheumatology 2008 recommendations for the use of nonbiologic and biologic disease-modifying antirheumatic drugs in rheumatoid arthritis. Arthritis Rheum 2008; 59:762-784.

50 Conway R, Low C, Coughlan RJ, et al: Methotrexate and lung disease in rheumatoid arthritis - a meta-analysis of randomized controlled trials. Arthritis Rheum 2014;66:803812.

-51 Alarcon GS, Kremer JM, Macaluso M, et al: Risk factors for methotrexate-induced lung injury in patients with rheumatoid arthritis. A multicenter, case-control study. Methotrexate-Lung Study Group. Ann Intern Med 1997; 127:356-364.

52 Suissa S, Hudson M, Ernst P: Leflunomide use and the risk of interstitial lung disease in rheumatoid arthritis. Arthritis Rheum 2006;54: 1435-1439.

53 Chikura B, Lane S, Dawson JK: Clinical expression of leflunomide-induced pneumonitis. Rheumatology (Oxford) 2009;48:10651068.

54 Sawada T, Inokuma S, Sato T, et al; Study Committee for Leflunomide-Induced Lung Injury, Japan College of Rheumatology: Leflunomide-induced interstitial lung disease: prevalence and risk factors in Japanese patients with rheumatoid arthritis. Rheumatology (Oxford) 2009;48:1069-1072.

55 Panopoulos ST, Sfikakis PP: Biological treatments and connective tissue disease associated interstitial lung disease. Curr Opin Pulm Med 2011;17:362-367.

56 Perez-Alvarez R, Perez-de-Lis M, DiazLagares $\mathrm{C}$, et al: Interstitial lung disease induced or exacerbated by TNF-targeted therapies: analysis of 122 cases. Semin Arthritis Rheum 2011;41:256-264.
Treatment Considerations of Lung Involvement in Rheumatologic Disease 
57 Dixon WG, Hyrich KL, Watson KD, et al: Influence of anti-TNF therapy on mortality in patients with rheumatoid arthritis-associated interstitial lung disease: results from the British Society for Rheumatology Biologics Register. Ann Rheum Dis 2010;69:1086-1091.

58 Herrinton LJ, Harrold LR, Liu L, et al: Association between anti-TNF- $\alpha$ therapy and interstitial lung disease. Pharmacoepidemiol Drug Saf 2013;22:394-402.

59 Robles-Perez A, Rodriguez-Sanchon B, Dorca $\mathrm{J}$, et al: Preclinical lung disease in early rheumatoid arthritis. Chron Respir Dis 2015, in press.

60 Imokawa S, Colby TV, Leslie KO, et al: Methotrexate pneumonitis: review of the literature and histopathological findings in nine patients. Eur Respir J 2000;15:373-381.

61 Kreider M, Highland K: Pulmonary involvement in Sjögren syndrome. Semin Respir Crit Care Med 2014;35:255-264.

62 Parambil JG, Myers JL, Lindell RM, et al: Interstitial lung disease in primary Sjögren syndrome. Chest 2006;130:1489-1495.

63 Cha SI, Fessler MB, Cool CD, et al: Lymphoid interstitial pneumonia: clinical features, associations and prognosis. Eur Respir J 2006;28: 364-369.

64 Deheinzelin D, Capelozzi VL, Kairalla RA, et al: Interstitial lung disease in primary Sjögren's syndrome. Clinical-pathological evaluation and response to treatment. Am J Respir Crit Care Med 1996;154:794-799.

-65 Gottenberg JE, Cinquetti G, Larroche C, et al; Club Rhumatismes et Inflammations and the French Society of Rheumatology: Efficacy of rituximab in systemic manifestations of primary Sjögren's syndrome: results in 78 patients of the AutoImmune and Rituximab registry. Ann Rheum Dis 2013;72:1026-1031.

-66 Swartz MA, Vivino FB: Dramatic reversal of lymphocytic interstitial pneumonitis in Sjögren's syndrome with rituximab. J Clin Rheumatol 2011;17:454.

67 Seror R, Sordet C, Guillevin L, et al: Tolerance and efficacy of rituximab and changes in serum B cell biomarkers in patients with systemic complications of primary Sjögren's syndrome. Ann Rheum Dis 2007;66:351-357.

68 Tan EM, Cohen AS, Fries JF, et al: The 1982 revised criteria for the classification of systemic lupus erythematosus. Arthritis Rheum 1982;25:1271-1277.
69 Winslow WA, Ploss LN, Loitman B: Pleuritis in systemic lupus erythematosus: its importance as an early manifestation in diagnosis. Ann Intern Med 1958;49:70-88.

-70 Muangchan C, van Vollenhoven RF, Bernatsky SR, et al: Treatment algorithms in systemic lupus erythematosus. Arthritis Care Res (Hoboken) 2015, Epub ahead of print.

71 Colby TV: Pulmonary pathology in patients with systemic autoimmune diseases. Clin Chest Med 1998;19:587-612.

72 Gutsche M, Rosen GD, Swigris JJ: Connective tissue disease-associated interstitial lung disease: a review. Curr Respir Care Rep 2012;1: 224-232.

73 Martínez-Martínez MU, Abud-Mendoza C: Recurrent diffuse alveolar haemorrhage in a patient with systemic lupus erythematosus: long-term benefit of rituximab. Lupus 2012; 21:1124-1127.

-74 Al Rashidi A, Alajmi M, Hegazi MO: Mycophenolate mofetil as a maintenance therapy for lupus-related diffuse alveolar hemorrhage: a case report. Lupus 2011;20:15511553.

75 Claridge S, Das P, Dorling A, et al: Plasmapheresis as a rescue therapy for systemic lupus erythematosus-associated diffuse alveolar haemorrhage. BMJ Case Rep 2011;2011: bcr0220113893.

76 Carmier D, Diot E, Diot P: Shrinking lung syndrome: recognition, pathophysiology and therapeutic strategy. Expert Rev Respir Med 2011;5:33-39.

77 Calderaro DC, Ferreira GA: Presentation and prognosis of shrinking lung syndrome in systemic lupus erythematosus: report of four cases. Rheumatol Int 2012;32:1391-1396.

-78 Carmier D, Marchand-Adam S, Diot P, et al: Respiratory involvement in systemic lupus erythematosus. Rev Mal Respir 2010;27:e66e78.

79 Peñacoba Toribio P, Córica Albani ME, Myos Pérez M, et al: Rituximab in the treatment of shrinking lung syndrome in systemic lupus erythematosus. Rheumatol Clin 2013;10:325327.

-80 Connors GR, Christopher-Stine L, Oddis CV, et al: Interstitial lung disease associated with the idiopathic inflammatory myopathies: what progress has been made in the past 35 years? Chest 2010;138:1464-1474.

81 Cottin V, Thivolet-Béjui F, Reynaud-Gaubert $\mathrm{M}$, et al: Interstitial lung disease in amyopathic dermatomyositis, dermatomyositis and polymyositis. Eur Respir J 2003;22:245-250.
82 Marie I, Hatron PY, Dominique S, et al: Shortterm and long-term outcomes of interstitial lung disease in polymyositis and dermatomyositis: a series of 107 patients. Arthritis Rheum 2011;63:3439-3447.

83 Mukae H, Ishimoto H, Sakamoto N, et al: Clinical differences between interstitial lung disease associated with clinically amyopathic dermatomyositis and classic dermatomyositis. Chest 2009;136:1341-1347.

84 Schnabel A, Reuter M, Biederer J, et al: Interstitial lung disease in polymyositis and dermatomyositis: clinical course and response to treatment. Semin Arthritis Rheum 2003;32: 273-284.

-85 Marie I, Josse S, Hatron PY, et al: Interstitial lung disease in anti-Jo-1 patients with antisynthetase syndrome. Arthritis Care Res (Hoboken) 2013;65:800-808.

-86 Lega JC, Reynaud Q, Belot A, et al: Idiopathic inflammatory myopathies and the lung. Eur Respir Rev 2015;24:216-238.

87 Kurita T, Yasuda S, Oba K, et al: The efficacy of tacrolimus in patients with interstitial lung diseases complicated with polymyositis or dermatomyositis. Rheumatology 2015;54:3944.

88 Mira-Avendano IC, Parambil JG, Yadav R, et al: A retrospective review of clinical features and treatment outcomes in steroid-resistant interstitial lung disease from polymyositis/ dermatomyositis. Respir Med 2013;107:890896.

89 Sem M, Molberg O, Lund MB, et al: Rituximab treatment of the anti-synthetase syndrome: a retrospective case series. Rheumatology (Oxford) 2009;48:968-971.

90 Vij R, Strek ME: Diagnosis and treatment of connective tissue disease-associated interstitial lung disease. Chest 2013;143:814-824.

-91 Simeon-Aznar CP, Fonollosa-Pla V, TolosaVilella C, et al: Effect of mycophenolate sodium in scleroderma-related interstitial lung disease. Clin Rheumatol 2011;30:1393-1398.

92 Meiners PM, Vissink A, Kroese FG, et al: Abatacept treatment reduces disease activity in early primary Sjögren's syndrome (openlabel proof of concept ASAP study). Ann Rheum Dis 2014;73:1393-1396.

93 Buch MH, Vital EM, Emery P: Abatacept in the treatment of rheumatoid arthritis. Arthritis Res Ther 2008;10(suppl 1):S5. 